\title{
Glutathione synthesis is compromised in erythrocytes from individuals with HIV
}

\author{
Devin Morris ${ }^{1}$, Judy Ly ${ }^{1}$, Po-Ting Chi ${ }^{2}$, John Daliva ${ }^{2}$, Truongson Nguyen ${ }^{2}$, Charleen Soofer ${ }^{2}$, \\ Yung C. Chen ${ }^{2}$, Minette Lagman ${ }^{2}$ and Vishwanath Venketaraman ${ }^{1}$ * \\ ' Department of Basic Medical Sciences, College of Osteopathic Medicine of the Pacific, Western University of Health Sciences, Pomona, CA, USA \\ ${ }_{2}^{2}$ Graduate College of Biomedical Sciences, Western University of Health Sciences, Pomona, CA, USA
}

\section{Edited by:}

Alfonso Pompella, Università di Pisa, Italy

\section{Reviewed by:}

Manuela Mengozzi, Brighton and Sussex Medical School, UK

Alfonso Pompella, Università di Pisa, Italy

\section{*Correspondence:}

Vishwanath Venketaraman

Department of Basic Medical

Sciences, College of Osteopathic

Medicine of the Pacific, Western

University of Health Sciences, 309

East Second Street, Pomona,

CA 91766, USA

e-mail:vvenketaraman@

westernu.edu
We demonstrated that the levels of enzymes responsible for the synthesis of glutathione (GSH) such as glutathione synthase (GSS), glutamate-cysteine ligase-catalytic subunit (GCLC), and glutathione reductase (GSR) were significantly reduced in the red blood cells (RBCs) isolated from individuals with human immunodeficiency virus (HIV) infection and this reduction correlated with decreased levels of intracellular GSH. GSH content in RBCs can be used as a marker for increased overall oxidative stress and immune dysfunctions caused by HIV infection. Our data supports our hypothesis that compromised levels of GSH in HIV infected individuals' is due to decreased levels of GSH-synthetic enzymes. The role of GSH in combating oxidative stress and improving the functions of immune cells in HIV patients' indicates the benefit of an antioxidant supplement which can reduce the cellular damage and promote the functions of immune cells.

Keywords: glutathione, HIV, GSS, GCL, GSR

\section{INTRODUCTION}

Roughly 34 million people around the world are infected with human immunodeficiency virus (HIV). Since its first reporting in 1981, the beginning of an epidemic, 60 million people have contracted HIV and an estimated 30 million have died due to HIV related causes (World Health Organization, 2010). HIV infection is associated with a wide range of different opportunistic infections that are usually the prime suspect for patients' poor survival. Among the array of opportunistic infections, one of the leading life-threatening infection common among HIV positive individuals with compromised immune system is Mycobacterium tuberculosis. Especially in developing countries, as many as eighty percent of people with AIDS are at risk of developing tuberculosis (TB) (World Health Organization, 2009). HIV's primary targets in vivo are blood monocytes, CD4 T lymphocytes, and resident macrophages. Due to HIV's high affinity for infecting and killing $\mathrm{CD} 4+\mathrm{T}$ lymphocytes, cell-mediated immunity is drastically lowered. This results in greater probability for opportunistic infections, primarily M. tuberculosis (Levy, 1993; Pantaleo et al., 1993; Droge and Holm, 1997; Herzenberg etal., 1997).

Glutathione (GSH) is a major component involved in the control and maintenance of cellular redox state and cellular homeostasis (Griffith, 1999). In addition, GSH is also important in an array of cellular functions such as protein synthesis, transport across membranes, receptor action, and cell growth (Griffith, 1999). As a natural antioxidant, GSH scavenges peroxide species. Low levels of GSH have been shown to play a role in the apoptosis of CD4+ T cells, which is the major pathology of the HIV infection, therefore signifying the importance of GSH (Levy, 1993;
Pantaleo et al., 1993; Droge and Holm, 1997; Herzenberg et al., 1997).

Glutathione is produced by almost all cell types and are present in two forms, reduced ( $r \mathrm{GSH}$ ) and oxidized (GSSG). $r \mathrm{GSH}$ is synthesized by two different mechanisms. De novo synthesis of $r \mathrm{GSH}$ occurs in a two-step process mediated by two different enzymes, glutamate-cysteine ligase (GCL) and glutathione synthase (GSS). $r$ GSH is also synthesized via the reduction of GSSG by glutathione reductase (GSR; Staal, 1998). In this study, we went beyond the innate immune response components and investigated the changes in the levels of GSH in red blood cells (RBCs) isolated from individuals with HIV infection. We hypothesized that compromised levels of GSH in HIV-infected individuals is due to decreased levels of enzymes that are involved in the synthesis of GSH. Since RBCs are systemically present in abundance, we tested our hypothesis by determining the extent to which the levels of GSH-synthetic enzymes are compromised in RBCs derived from individuals with HIV infection and correlating decreased levels of GSH-synthetic enzymes with deficiency in the levels of GSH.

\section{MATERIALS AND METHODS SUBJECTS}

The protocol was approved by Institutional Review Board with the requirement that each volunteer recruited would need to be given a consent form that described the basis and the procedures of the study. A signed informed consent from each volunteer that agreed to participate was obtained. A total of 16 volunteers (eight healthy subjects and eight individuals with HIV infection) were recruited for the study. Individuals with HIV infection were recruited from the Foothills AIDS project. Healthy subjects without HIV infection 
or a history of TB were recruited from the staff of Western University of Health Sciences. All HIV-infected volunteers had been diagnosed with HIV-1, were taking some form of anti-retroviral treatment, and had CD4+ T-cell counts between 271 and 1415 cells per $\mathrm{mm}^{3}$. Thirty five milliliters $(\mathrm{mL})$ of blood was drawn once from both healthy volunteers and individuals with HIV infection.

\section{ERYTHROCYTE ISOLATION}

Red blood cells were isolated from whole blood by density gradient centrifugation with FICOLL-Paque (GE Healthcare, 17-440-02). RBCs that aggregated as the bottom layer were collected and stored at $-20^{\circ} \mathrm{C}$ in a cell lysis/protein storage buffer [ $20 \mathrm{mM}$ Tris, $100 \mathrm{mM}$ $\mathrm{NaCl}$ (Amresco, N653), 1X protease inhibitor cocktail (Amresco, M221)] for western blot analysis.

\section{GEL ELECTROPHORESIS AND WESTERN BLOT ANALYSIS IN RBC LYSATES FROM HEALTHY AND HIV+ SUBJECTS}

Total protein content was determined using Coomassie blue colorimetric assay (Thermo Scientific, PI-23200). $200 \mu \mathrm{g}$ of total RBC proteins per sample were separated via denaturing polyacrylamide electrophoresis (12\%). Separated proteins were transferred to a Polyvinylidene fluoride membrane (GE, PV4HY00010) by electroblotting. Membranes were blocked for $1 \mathrm{~h}$ at room temperature in tris buffered saline with tween 20 (TBST) and $5 \%$ non-fat dry milk followed by three washes (15 min for each wash) in TBST with mild shaking. The membranes were then incubated with a primary antibody overnight at $4^{\circ} \mathrm{C}$ in TBST with gentle shaking. The primary antibodies used were mouse anti-human GSS (1:1000, Abcam, ab5513), mouse antihuman GSR (1:500, Abcam, ab55075), and rabbit anti-human glutamate-cysteine ligase-catalytic subunit (GCLC; 1:250, Abcam, ab40929). Following overnight incubation with the primary antibodies, membranes were washed five times for $15 \mathrm{~min}$ in TBST with mild shaking. Washed membranes were incubated with a secondary antibody conjugated with horse radish peroxidase, anti-mouse (1:1000, Abcam, ab7064), or anti-rabbit (1:1000, Abcam, ab72465) in TBST for an hour at room temperature. Membranes were washed again five times for $15 \mathrm{~min}$ in TBST with mild shaking. Chemiluminescent substrate was applied to the membranes which were then exposed to an $\mathrm{x}$ ray film (Genemate, F-9024) and developed in a dark room. Digital images of the immunoblots were captured using a Versadoc gel imaging system (Bio-rad, 4000 MP). Densitometric analysis of the images was performed using ImageJ, a free software program available from the National Institutes of Health (http://rsbweb.nih.gov/ij/).

\section{ASSAY OF GSH LEVELS IN RBCs FROM HEALTHY AND HIV+ SUBJECTS}

Glutathione concentrations were measured in RBCs isolated from healthy and $\mathrm{HIV}^{+}$individuals by spectrophotometry using a colorimetric assay kit (Arbor Assays, K006-H1). RBCs were suspended in an ice cold 5\% 5-sulfosalicylic acid dihydrate solution (MP Biomedicals, 160001-4924H). Supernatants collected after centrifugation were analyzed for the total GSH as per the manufacturer's instructions. All GSH measurements were normalized with total protein concentrations.

\section{STATISTICAL ANALYSIS}

Statistical analysis of the data was carried out using GraphPad Prism 6 . The data was analyzed by comparing the means of $n=8$ individuals (unless otherwise specified) using unpaired student's $t$-tests. $P \leq 0.05$ was considered statistically significant.

\section{RESULTS AND DISCUSSION}

Glutathione is a tripeptide made of glutamine, cysteine, and glycine. In the de novo synthesis of GSH, glutamine is linked to cysteine by GCL to form -glutamylcysteine (Griffith, 1999). Then GSS links the dipeptide -glutamylcysteine to glycine to form the final GSH molecule (Griffith, 1999). The GSH redox system plays a major role in ridding the body of oxidative stress and restoring homeostasis (Griffith, 1999). To elicit antioxidant effects, GSH is converted to oxidized glutathione (GSSG) by glutathione peroxidase (GPx). GSSG can be converted back to GSH by GSR (Staal, 1998). It is important to note that only free GSH has antioxidant effects. On the other hand, GSSG lacks antioxidant functions and is a byproduct of the scavenging activity of GSH (Staal, 1998; Griffith, 1999). GSH/GSSG ratio should be maintained to optimize the GSH redox system. GCL, the rate-limiting enzyme of GSH synthesis, is composed of a catalytic subunit (GCLC) and a modulating subunit (GCLM). GCLC is the component that performs the amino acid linkage between glutamine and cysteine, whereas GCLM modulates the activity of GCLC (Huang et al., 1993).

It has previously been reported that GSH levels in the plasma, erythrocytes, and peripheral blood mononuclear cells (PBMC) of $\mathrm{HIV}^{+}$individuals are compromised (Sbrana et al., 2004; Venketaraman etal., 2006; Guerra etal., 2011, 2012; Morris et al., 2012, 2013). The goal of our study is to characterize the causes for diminished levels of GSH in HIV infected individuals by determining the extent to which the levels of GCLC, GSS, and GSR are decreased in RBCs isolated from individuals with HIV infection compared to healthy subjects. Measurement of GSS and GCLC revealed a significant decrease in the levels of these enzymes present in RBCs of HIV-infected individuals compared to healthy subjects (Figures 1 and 2). Both GSS and GCLC are crucial enzymes that are involved in the catalytic rate limiting step and second step reaction, respectively, in the biosynthesis of GSH (Staal, 1998; Griffith, 1999; Morris et al., 2012, 2013). We also observed a significant decrease in the expression of GSR in RBCs isolated from HIV positive subjects (Figure 3). This explains the reason for decreased levels of GSH and the consequences related to the GSH deficiency such as loss of immune function observed in HIV patients (Venketaraman et al., 2006; Guerra etal., 2011, 2012; Morris etal., 2012, 2013). Reduced expressions of GSH synthesis enzymes in RBCs from individuals with HIV infection was accompanied by decreased levels of total GSH (Figure 4).

$\mathrm{HIV}^{+}$individuals were also found to have increased levels of TGF- $\beta$ in their plasma and macrophage supernatants (Morris et al., 2012). Moreover, TGF- $\beta$ is known to block the production of GCLC which leads to decreased GSH synthesis (Morris etal., 2012). HIV-1 transactivator protein (TAT) decreases the amount of GSH present in mice through the modulation of GSH biosynthetic enzymes (Choi et al., 2000). 
A

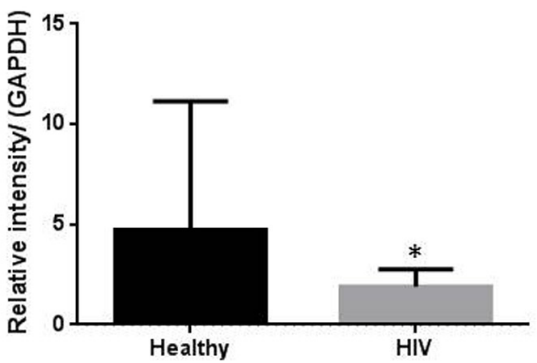

$91 \mathrm{kDa}$

\section{$52 \mathrm{kDa}$}

$48 \mathrm{kDa}$
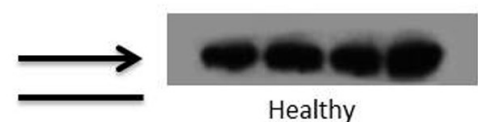

$38 \mathrm{kDa}$

$34 \mathrm{kDa}$


GAPDH

FIGURE 1 | Quantifying of GSS enzyme levels in Healthy and HIV-infected subjects. Red blood cell samples were separated from blood of healthy volunteers and HIV-infected individuals were used for measurement of GSS, the enzyme that is involved in the second step of GSH synthesis. Electrophoresis and Western blot were used. There was a significant decrease of GSS in the RBC of HIV-infected individuals compared to healthy individuals. Data in (A) represents significant difference in the levels of GSS between RBCs isolated from eight healthy individuals and eight individuals with HIV infection $\left({ }^{*} p \leq 0.05\right)$. (B): Illustrates representative images of GSS and their corresponding GAPDH bands from four healthy subjects and four individuals with HIV infection.

\section{GCLC}

A

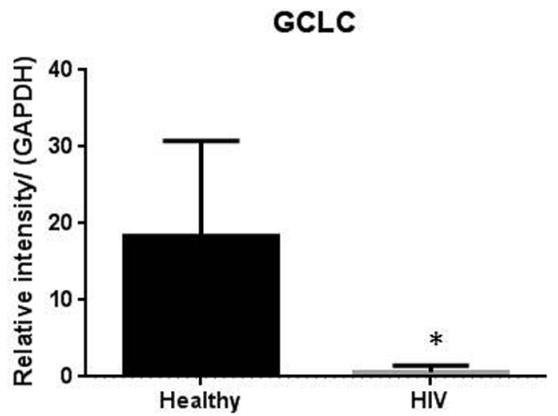

B

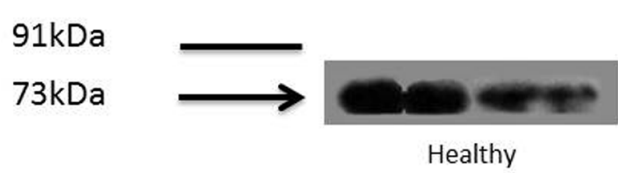

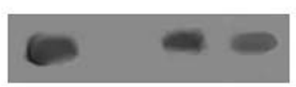

HIV

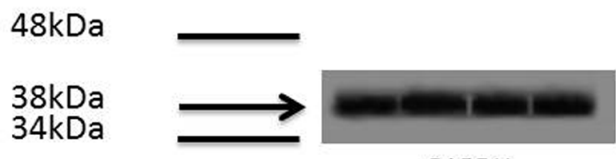

GAPDH

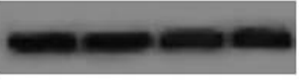

GAPDH
FIGURE 2 | Quantifying of GCLC enzyme levels in Healthy and HIVinfected subjects. Red blood cell samples were separated from blood of healthy volunteers and HIV-infected individuals were used for measurement of the catalytic subunit of GCLC that is responsible for the linkage between glutamine and cysteine in the first step of GSH synthesis. Electrophoresis and Western blot were used. GCLC levels were significantly lower in the
RBC of HIV-infected individuals compared to healthy subjects. Data in (A) represents significant difference in the levels of GCLC between RBCs isolated from eight healthy individuals and eight individuals with HIV infection $\left.{ }^{*} p \leq 0.05\right)$. (B): Illustrates representative images of GCLC and their corresponding GAPDH bands from four healthy subjects and four individuals with HIV infection. 


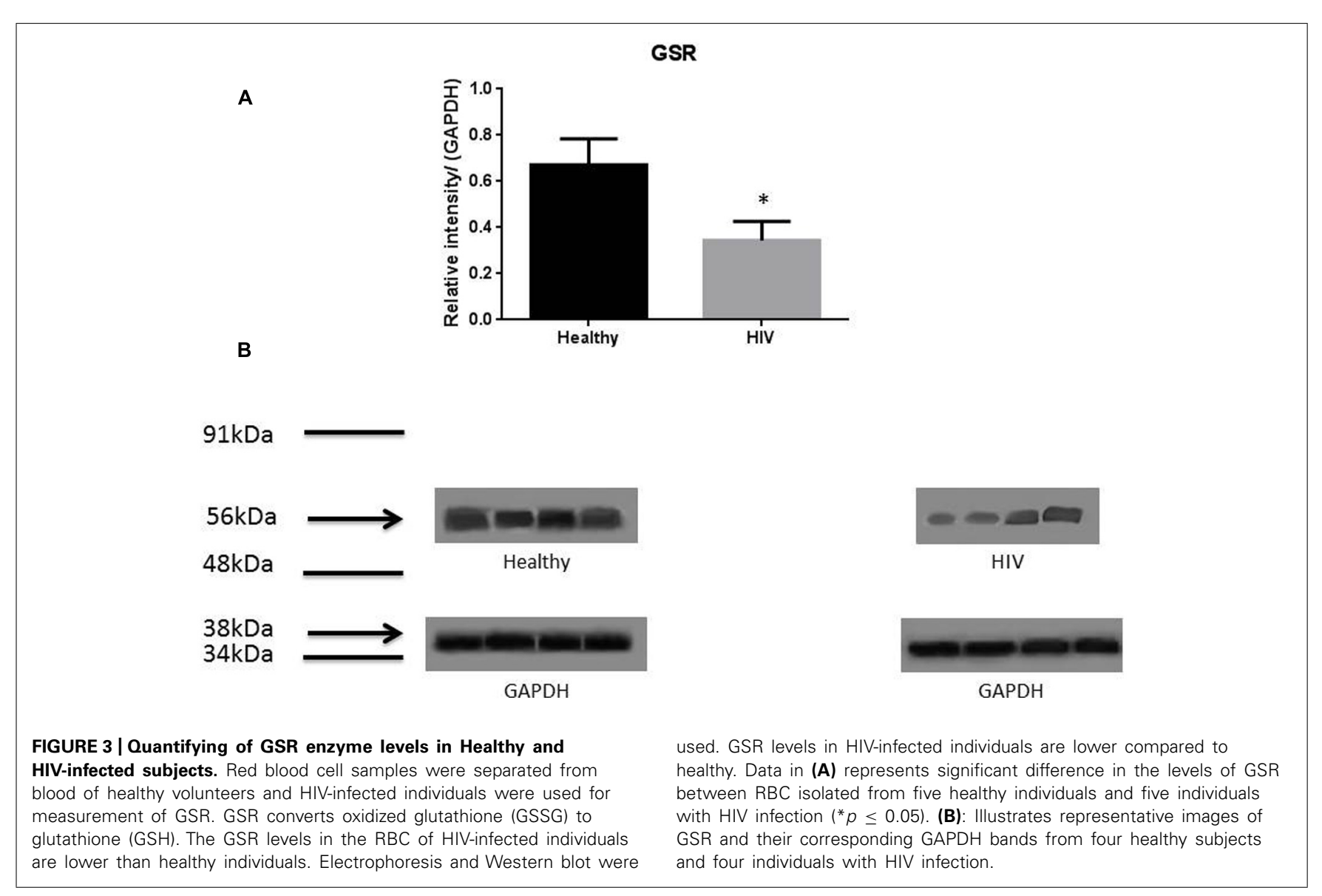

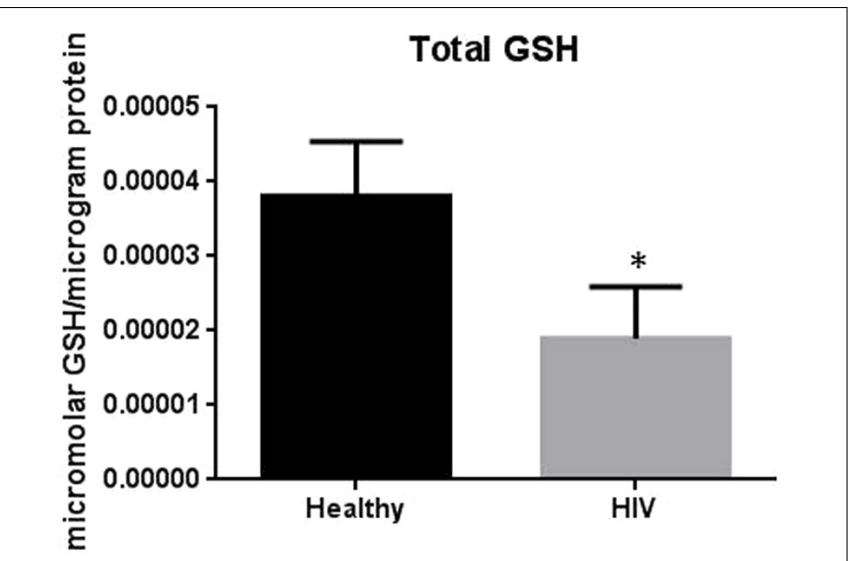

FIGURE 4 | Assay of GSH levels in RBCs from healthy subjects and individuals with HIV infection. Analysis of total GSH which includes both free GSH and GSSG indicated significantly higher levels of GSH in healthy individuals compared to HIV positive subjects. Data in Figure $\mathbf{4}$ represents significant difference in the levels of total GSH between RBCs isolated from five healthy individuals and five individuals with HIV infection ( ${ }^{*} p=0.05$ ).

TAT also increases free radical production. Therefore, marked increase in oxidative stress along with increased levels of TGF$\beta$ lead to the compromised levels of GSH synthesis enzymes. The master transcription factor nuclear factor (erythroid-derived 2)-like 2 (Nrf2) regulates the expression of antioxidant and phase II-metabolizing enzymes by activating the antioxidant response element (ARE) and thereby protects cells and tissues from oxidative stress. The Nrf2 gene binding to the ARE results in the upregulation of GSH synthesis enzymes such as GCLC, GCLM, and GSR. New findings argue that HIV-1-related proteins downregulate $\mathrm{Nrf2}$ expression and/or activity within the alveolar epithelium, which in turn impairs antioxidant defenses and barrier function, thereby rendering the lung susceptible to oxidative stress and injury (Fan etal., 2013). Furthermore, this study suggests that activating the Nrf2/ARE pathway with the dietary supplement sulforaphane could augment antioxidant defenses and lung health inHIV-1-infected individuals (Fan et al., 2013).

We have previously reported that the virulent laboratory strain of M. tuberculosis $\mathrm{H} 37 \mathrm{Rv}$ is sensitive to GSH at physiological concentrations $(5 \mathrm{mM})$ when grown in vitro (Venketaraman et al., 2005). Thus, GSH has direct antimycobacterial activity, functioning as an effector molecule in innate defense against $M$. tuberculosis infection (Venketaraman et al., 2005; Dayaram et al., 2006). We recently reported that GSH is integral in facilitating the control of intracellular growth of M. tuberculosis in human macrophages (Venketaraman etal., 2005; Dayaram etal., 2006; Morris et al., 2012, 2013). These results further confirm that GSH has direct antimycobacterial activity and unfolds a novel and potentially important innate defense mechanism adopted by 


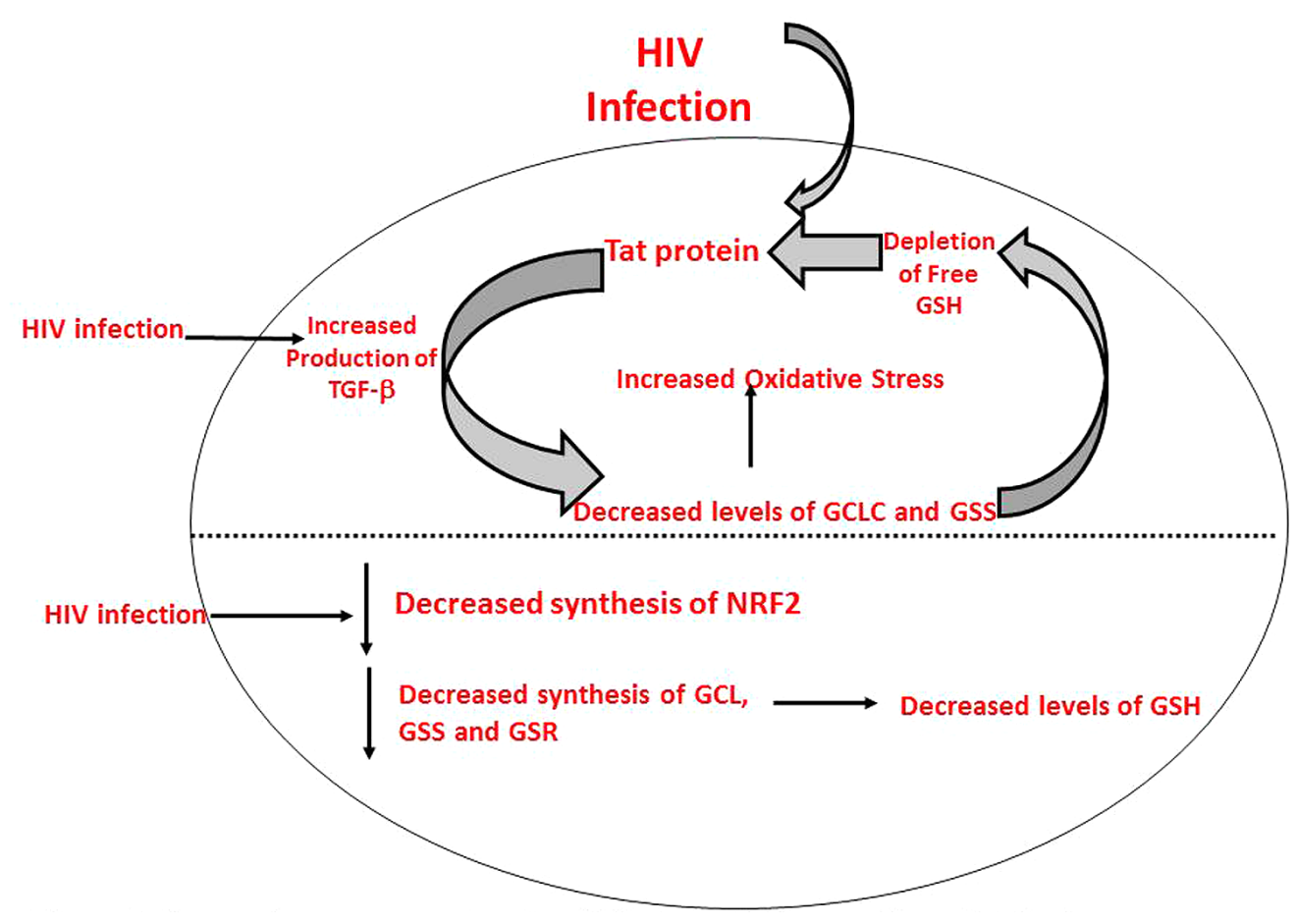

FIGURE 5 | Model illustrating causes for decreased levels of GSH in individuals with HIV infection.

human macrophages to control M. tuberculosis infection. We also demonstrated that GSH in combination with cytokines such as IL-2 and IL-12 enhances the functional activity of natural killer (NK) cells to inhibit the growth of M. tuberculosis inside human monocytes (Millman et al., 2008; Guerra et al., 2012). Importantly, data from our most recent studies indicate that GSH activates the functions of T lymphocytes to control M. tuberculosis infection inside human monocytes (Guerra et al., 2011). These results indicate that GSH inhibits the growth of $M$. tuberculosis by both direct antimycobacterial effects as well as by activating the functions of immune cells (Venketaraman et al., 2005; Dayaram etal., 2006; Millman et al., 2008; Guerra et al., 2011, 2012; Morris et al., 2012, 2013). We also reported that the GSH concentrations were significantly lower in macrophages, NK, and T cells isolated from individuals with HIV infection compared to healthy subjects (Venketaraman et al., 2006; Guerra et al., 2011, 2012; Morris etal., 2012, 2013). Decreased levels of GSH in macrophages, NK , and T cells derived from individuals with HIV infection was accompanied by diminished control of intracellular M. tuberculosis infection (Venketaraman et al., 2006; Guerra et al., 2011, 2012; Morris et al., 2012,2013). Our group is a pioneer in reporting that GSH levels were decreased in macrophages, $\mathrm{T}$ cells, and NK cells from individuals with HIV infection and correlating decreased GSH levels with impaired innate and adaptive immune responses against $M$. tuberculosis infection (Venketaraman etal., 2006; Guerra et al., 2011, 2012; Morris et al., 2012, 2013).

In this study we investigated the cause for decreased levels of GSH in individuals with HIV infection by quantifying the levels of
GSS, GCLC, and GSR in the RBCs derived from healthy subjects and individuals with HIV infection. The results of the Western Blot indicate that there is a significant difference in the levels of GSS, GCLC, and GSR between HIV-infected individuals and healthy individuals, which supports our hypothesis that individuals with HIV infection have lower concentrations of enzymes that are responsible for both de novo synthesis of GSH and conversion of GSSG to GSH (Figures 1-3). In addition, our results indicate that there is a significant decrease in the levels of total GSH in the RBCs derived from HIV-infected individuals (Figure 4). Overall, these significant findings indicating lower levels of GSS, GCLC, and GSR in HIV-infected individuals support our hypothesis and contribute to previous findings that there are lower levels of GSH in HIV-infected individuals than healthy individuals (Figure 5). Observations from the current study combined with our previous findings strongly suggest that liposomal formulations of GSH can be used as a possible supplement to current HIV treatments since they can provide complete $r$ GSH molecules, bypassing the cellular machinery for GSH production. Liposomal formulations containing GSH can be more effective in supplementing the intracellular $r \mathrm{GSH}$ and restoring the immune cell functions including the antimycobacterial activity in macrophages from HIV patients at concentrations lower than NAC (Venketaraman et al., 2006; Guerra et al., 2011, 2012; Morris et al., 2012, 2013).

\section{ACKNOWLEDGMENTS}

We would like to acknowledge Dr. Cesar Ochoa for helping us with the blood draw for the study. We would like to thank all of 
our volunteers for without them this study would not have been possible.

\section{REFERENCES}

Choi, J., Liu, R. M., Kundu, R. K., Sangiorgi, F., Wu, W., Maxson, R., et al. (2000). Molecular mechanism of decreased glutathione content in human immunodeficiency virus type 1 Tat-transgenic mice. J. Biol. Chem. 275, 3693-3698. doi: 10.1074/jbc.275.5.3693

Dayaram, Y. K., Talaue, M. T., Connell, N. D., and Venketaraman, V. (2006). Characterization of a glutathione metabolic mutant of Mycobacterium tuberculosis and its resistance to glutathione and nitrosoglutathione. J. Bacteriol. 188, 1364-1372. doi: 10.1128/JB.188.4.1364-1372.2006

Droge, W., and Holm, E. (1997). Role of cysteine and glutathione in HIV infection and other diseases associated with muscle wasting and immunological dysfunction. FASEB J. 11, 1077-1089.

Fan, X., Staitieh, B. S., Jensen, J. S., Mould, K. J., Greenberg, J. A., Joshi, P. C., et al. (2013). Activating the Nrf2-mediated antioxidant response element restores barrier function in the alveolar epithelium of HIV-1 transgenic rats. Am. J. Physiol. Lung Cell. Mol. Physiol. 305, L267-L277. doi: 10.1152/ajplung.002 88.2012

Griffith, O. W. (1999). Biologic and pharmacologic regulation of mammalian glutathione synthesis. Free Radic. Biol. Med. 27, 922-935. doi: 10.1016/S08915849(99)00176-8

Guerra, C., Johal, K., Morris, D., Moreno, S., Alvarado, O., Gray, D., et al. (2012). Control of Mycobacterium tuberculosis growth by activated natural killer cells. Clin. Exp. Immunol. 168, 142-152. doi: 10.1111/j.1365-2249.2011.04552.x

Guerra, G., Morris, D., Gray, D., Tanzil, M., Sipin, A., and Kung, S. (2011) Adaptive immune responses against Mycobacterium tuberculosis infection in healthy and HIV infected individuals. PLOS ONE 6:e28378. doi: 10.1371/journal.pone. 0028378

Herzenberg, L. A., De Rosa, S. C., Dubs, J. G., Roederer, M., Anderson, M. T. Ela, S. W., et al. (1997). Glutathione deficiency is associated with impaired survival in HIV disease. Proc. Natl. Acad. Sci. U.S.A. 94, 1967-1972. doi 10.1073/pnas.94.5.1967

Huang, C. S., Chang, L. S., Anderson, M. E., and Meister A. (1993). Catalytic and regulatory properties of the heavy subunit of rat kidney $\gamma$-glutamylcysteine synthase. J. Biol. Chem. 268, 19675-19680.

Levy, J. A. (1993). HIV pathogenesis and long term survival. AIDS 7, 1401-1410. doi: 10.1097/00002030-199311000-00001

Millman, A. C., Salman, M., Dayaram, Y. K., Connell, N. D., and Venketaraman, V. (2008). Natural killer cells, glutathione, cytokines, and innate immunity against Mycobacterium tuberculosis. J. Interferon Cytokine Res. 28, 153-165. doi: 10.1089/jir.2007.0095

Morris, D., Guerra, C., Donohue, C., Oh, H., Khurasany, M., and Venketaraman, V. (2012). Unveiling the mechanisms for decreased glutathione in individuals with HIV infection. Clin. Dev. Immunol. 2012:734125. doi: 10.1155/2012/734125
Morris, D., Guerra, C., Khurasany, M., Guilford, F., Saviola, B., Huang, Y., et al. (2013). Glutathione supplementation improves macrophage functions in HIV. J. Interferon Cytokine Res. 33, 270-279. doi: 10.1089/jir. 2012.0103

Pantaleo, G., Graziosi, C., and Fauci, A. S. (1993). New concepts in the immunopathogenesis of human immuno deficiency infection. N. Engl. J. Med. 328, 327-335. doi: 10.1056/NEJM199302043280508

Sbrana, E., Paladini, A., Bramanti, E., Spinetti, M. C., and Raspi, G. (2004). Quantitation of reduced glutathione and cysteine in human immunodefiency virus-infected patients. Electrophoresis 25, 1522-1529. doi: 10.1002/elps.200 305848

Staal, F. J. (1998). Glutathione and HIV infection: reduced reduced or increased oxidized? Eur. J. Clin. Invest. 2, 194-196. doi: 10.1046/j.1365-2362.1998. 00268.x

Venketaraman, V., Dayaram, Y. K., Talaue, M. T., and Connell, N. D. (2005). Glutathione and nitrosoglutathione in macrophage defense against Mycobacterium tuberculosis. Infect. Immun. 73, 1886-1889. doi: 10.1128/IAI.73.3.1886-1889.2005

Venketaraman, V., Rodgers, T., Linares, R., Reilly, N., Swaminathan, S., Hom, D., et al. (2006). Glutathione and growth inhibition of Mycobacterium tuberculosis in healthy and HIV infected subjects. AIDS Res. Ther. 3:5. doi: 10.1186/17426405-3-5

World Health Organization. (2009). Global Tuberculosis Control: Surveillance, Planning and Financing. WHO Report (WHO/HTM/TB/2009.411). Geneva: World Health Organization.

World Health Organization. (2010). Towards Universal Access: Scaling up Priority HIV/AIDS Interventions in the Health Sector. Progress Report 2010. Geneva: World Health Organization.

Conflict of Interest Statement: The authors declare that the research was conducted in the absence of any commercial or financial relationships that could be construed as a potential conflict of interest.

Received: 03 March 2014; paper pending published: 19 March 2014; accepted: 28 March 2014; published online: 11 April 2014.

Citation: Morris D, Ly J, Chi P-T, Daliva J, Nguyen T, Soofer C, Chen YC, Lagman $M$ and Venketaraman $V$ (2014) Glutathione synthesis is compromised in erythrocytes from individuals with HIV. Front. Pharmacol. 5:73. doi: 10.3389/fphar.2014.00073

This article was submitted to Experimental Pharmacology and Drug Discovery, a section of the journal Frontiers in Pharmacology.

Copyright (c) 2014 Morris, Ly, Chi, Daliva, Nguyen, Soofer, Chen, Lagman and Venketaraman. This is an open-access article distributed under the terms of the Creative Commons Attribution License (CC BY). The use, distribution or reproduction in other forums is permitted, provided the original author(s) or licensor are credited and that the original publication in this journal is cited, in accordance with accepted academic practice. No use, distribution or reproduction is permitted which does not comply with these terms. 